\title{
Automorphism Group and Other Properties of Zero Component Graph over a Vector Space
}

\author{
Shikun Ou ${ }^{1},{ }^{1}$ Yanqi Fan, ${ }^{1}$ and Qunfang $\mathrm{Li}^{2}$ \\ ${ }^{1}$ School of Science, Jiangxi University of Science and Technology, Ganzhou, China \\ ${ }^{2}$ Department of Mathematics, Ganzhou Teachers College, Ganzhou, China
}

Correspondence should be addressed to Shikun Ou; ok6096@cumt.edu.cn

Received 20 January 2021; Revised 20 March 2021; Accepted 24 March 2021; Published 10 April 2021

Academic Editor: Elena Guardo

Copyright (c) 2021 Shikun Ou et al. This is an open access article distributed under the Creative Commons Attribution License, which permits unrestricted use, distribution, and reproduction in any medium, provided the original work is properly cited.

In this paper, we introduce an undirected simple graph, called the zero component graph on finite-dimensional vector spaces. It is shown that two finite-dimensional vector spaces are isomorphic if and only if their zero component graphs are isomorphic, and any automorphism of a zero component graph can be uniquely decomposed into the product of a permutation automorphism and a regular automorphism. Moreover, we find the dominating number, as well as the independent number, and characterize the minimum independent dominating sets, maximum independent sets, and planarity of the graph. In the case that base fields are finite, we calculate the fixing number and metric dimension of the zero component graphs and determine vector spaces whose zero component graphs are Hamiltonian.

\section{Introduction}

A binary relation on a vector space, group, or ring can be studied with the help of considering the associating graph defined by this relation. Symplectic graphs, orthogonal graphs, subspace inclusion graphs, and nonzero component graphs are such examples which have been considered recently (see $[1-4]$ ).

In this paper, we always assume $n \geq 2, F$ is a field, $F^{*}=F \backslash\{0\}$, and $\mathbb{V}$ is an $n$-dimensional vector space over $F$. Let $A=\left\{\varepsilon_{1}, \ldots, \varepsilon_{n}\right\}$ be a basis of $\mathbb{V}$. Then, any $\alpha \in \mathbb{V}$ can be uniquely written as $\alpha=\sum_{i=1}^{n} x_{i} \varepsilon_{i}$ with $x_{i} \in F$, and $S_{A}(\alpha)$ (resp., $\mathscr{Z}_{A}(\alpha)$ ) is the set of all basis vectors whose coefficients are nonzero (resp., zero) in the linear expression of $\alpha$ with respect to (i.e., with respect to) $A$, namely,

$$
\begin{aligned}
\mathcal{S}_{A}(\alpha) & =\left\{\varepsilon_{i} \mid \text { if } x_{i} \neq 0\right\}, \\
\text { (resp., } \mathscr{Z}_{A}(\alpha) & \left.=\left\{\varepsilon_{i} \mid \text { if } x_{i}=0\right\}\right) .
\end{aligned}
$$

Clearly, $S_{A}(\alpha) \cup \mathscr{Z}_{A}(\alpha)=A$ and $S_{A}(\alpha) \cap \mathscr{Z}_{A}(\alpha)=\varnothing$.

All graphs considered in this paper are undirected simple graphs (without loops and multiedges). The nonzero component graph $\Gamma_{c}(\mathbb{V})$ of $\mathbb{V}$, introduced by Das [1], is a graph with vertex set $\mathbb{V}^{*}=\mathbb{\bigvee}\{0\}$, in which for distinct $\alpha, \beta \in \mathbb{V}^{*}$, there exists an edge joining $\alpha$ and $\beta$ if and only if $\mathcal{S}_{A}(\alpha) \cap \mathcal{S}_{A}(\beta) \neq \varnothing$. In [1], the author studied the diameter, dominating number, and dependent number of $\Gamma_{c}(\mathbb{V})$. When the base field $F$ is finite, Das [5] considered the clique number, edge connectivity, and chromatic number of $\Gamma_{c}(\mathbb{V})$ and showed that $\Gamma_{c}(\mathbb{V})$ is Hamiltonian, but not Eulerian. Murtaza et al. [6] considered two parameters, called the locating-dominating number and identifying number, of $\Gamma_{c}(\mathbb{V})$. Also, the nonzero component union graph $\Gamma_{u}(\mathbb{V})$ of $\mathbb{V}$ has $\mathbb{V}^{*}$ as its vertex set, but two vertices $\alpha, \beta \in \mathbb{V}^{*}$ are adjacent if and only if $\left\langle\mathcal{S}_{A}(\alpha), \mathcal{S}_{A}(\beta)\right\rangle=\mathbb{V}$, where $\langle\mathcal{\delta}\rangle$ stands for the subspace of $\mathbb{V}$ generated by a subset $\delta \subseteq \mathbb{V}$. In [7], Das studied some properties of $\Gamma_{u}(\mathbb{V})$, such as the connectedness, dominating number as well as maximal cliques and showed that $\Gamma_{u}(\mathbb{V})$ is weakly perfect when the base field $F$ is finite.

It is well known that the automorphism group of a graph reflects the symmetry of the graph. Generally, it is an important but difficult work to describe the full automorphism group both in graph theory and in algebra. In [1], the author characterizes the automorphisms of $\Gamma_{c}(\mathbb{V})$. For the automorphisms of symplectic graphs, orthogonal graphs, and subspace inclusion graphs, the reader is referred to $[3,4,8]$. 
The fixing number and metric dimension of a graph (see Section 2 for the definitions) are two parameters which "destroy" the automorphisms and symmetry. Moreover, the fixing number has been used to consider the problem of programming a robot to manipulate objects (see [9]), while the metric dimension has been applied to various areas, such as pharmaceutical chemistry, robot navigation, diverse as combinatorial optimization, and sonar (see [10]). Recently, several authors considered the fixing number and metric dimension of some associating graphs defined on a vector space. Fazil [11] computed the fixing number of $\Gamma_{c}(\mathbb{V})$, and Benish [12] studied the relation between fixing number and another parameter, the fixed number, of $\Gamma_{c}(\mathbb{V})$. Ali et al. [13] calculated the metric dimension of $\Gamma_{c}(\mathbb{V})$. For a positive integer $k<n$, the Kneser graph $K(n, k)$ is a graph with all $k$-subsets of $[n]=\{1, \ldots, n\}$ as vertex set, in which two distinct $k$-subsets are connected by an edge if they are disjoint. Also, the vertex set of the Johnson graph $J(n, k)$ consists of all $k$-subsets of [ $n]$, but two distinct $k$-subsets are adjacent if their intersection is a $(k-1)$-subset. In [14], Boutin obtained sharp bounds for the fixing number of Kneser graphs and determined all Kneser graphs whose fixing number is 2, 3, or 4. In [15], using various algebraic, combinatorial, and geometric approaches, Bailey et al. studied the constructions of resolving sets of Kneser and Johnson graphs and provided bounds on their metric dimension. For more works on the fixing number and metric dimension, the reader is referred to $[9,16-21]$ and the references therein.

If $A$ is a basis of $\mathbb{V}$, then it is clear that $\mathbb{V}^{*}$ can be partitioned into $n$ classes:

$$
\mathscr{Z}_{j}(A)=\left\{\alpha \in \mathbb{V}|| \mathscr{Z}_{A}(\alpha) \mid=j\right\}, \quad 0 \leq j \leq n-1 .
$$

On the zero component graph $\Gamma(\mathbb{V}, A)$ of $\mathbb{V}$ with respect to $A$, we mean it is a graph defined as follows: $\Gamma(\mathbb{V}, A)$ has $\mathbb{V}_{*}(A)=\mathbb{V}^{*} \backslash \mathscr{Z}_{0}(A)$ as its vertex set, in which distinct $\alpha, \beta \in \mathbb{V}_{*}(A)$ are adjacent if and only if $\mathscr{Z}_{A}(\alpha) \cap \mathscr{Z}_{A}(\beta) \neq \varnothing$. Motivated by all the above, we intend to investigate the properties of $\Gamma(\mathbb{V}, A)$.

The rest of this paper is structured as follows: in Section 2, we introduce some definitions and notation in graph theory. In Section 3, we show that the zero component graph does not depend on the choice of the bases and thus restate the definition of the zero component graph (see Definition 1). In Section 4, we prove that two finite-dimensional vector spaces are isomorphic if and only if their zero component graphs are isomorphic. The automorphisms of the zero component graph are determined in Section 5, and the fixing number and metric dimension are computed in Section 6. Finally, we consider other properties of the zero component graph, such as the girth, minimum independent dominating sets, maximum independent sets, planarity, and hamiltonicity in Section 7.

\section{Notation and Preliminaries}

Let $G$ be a graph with a vertex set $V(G)$. Two vertices $x, y \in V(G)$ are said to be adjacent and written as $x \sim y$ if there exists an edge joining $x$ and $y$, otherwise, nonadjacent and $u * v$. A graph is said to be complete if any pair of its vertices are adjacent; as usual, $K_{n}$ stands for the complete graph of order $n$. Let $N(x)$ (resp., $\operatorname{deg}(x)$ ) be the neighbors (resp., degree) of $x$, that is, $N(x)=\{z \in V(G) \mid x \sim z\}$ (resp., $\operatorname{deg}(x)=|N(x)|)$. If $N(x)=N(y)$ or $N(x) \cup\{x\}=N$ $(y) \cup\{y\}$; then, $x$ and $y$ are said to be twins. We use Tw $(x)$ to denote the twin set of $x$, which consists of all twins of $x$ in $G$. In particular, a vertex $x \in V(G)$ is called a singleton whenever $\operatorname{Tw}(x)=\{x\}$. Clearly, the relation " $\approx$ " is equivalent if it is defined as $x \approx y \Leftrightarrow x \in \operatorname{Tw}(y)$; and then $V(G)$ is a union of some disjoint twin sets of $G$. The number of twin sets of $G$ is written as $\operatorname{tw}(G)$.

For $S \subseteq V(G)$, it is called a dominating set of $G$ if any vertex in $V(G) \backslash S$ is adjacent to at least one vertex in $S$, and it is called an independent set of $G$ if any pair vertices in $S$ are nonadjacent in $G$. Also, a dominating set is called an independent dominating set if it is an independent set. The minimum dominating set (resp., maximum independent set, minimum independent dominating set) of $G$ is a dominating set (resp., independent set, independent dominating set) with the minimum (resp., maximum, minimum) cardinality, and the cardinality of a minimum dominating set (resp., maximum independent set) is called the dominating number (resp., independent number) of $G$.

A trail in $G$ is an alternating sequence of vertices and edges, $x_{0} \sim x_{1} \sim \cdots \sim x_{k}$ for some positive integer $k$, where $x_{i}$ 's are not necessarily distinct; it is called a path if $x_{i}$ 's are distinct except for possibly the first and last vertices; and this path (resp., trail) is said to be joining $x_{0}$ and $x_{k}$. A graph is called connected if, for any pair of vertices, there exists a path joining them; it is called $k$-connected if each induced subgraph obtained by the graph from deleting $k-1$ vertices is connected. The connectivity of $G$ is the maximum value of $k$ for which $G$ is $k$-connected. A cycle of length $k$ is a path $x_{0} \sim x_{1} \sim \cdots \sim x_{k}$ with $x_{0}=x_{k}$; also, a cycle of length 3 is called a triangle. The girth of $G$ is the minimum of the lengths of all cycles in $G$. Moreover, a graph is called Eulerian (resp., Hamiltonian) if there exists a trail (resp., cycle) which contains all edges (resp., all vertices) in it exactly once.

For graphs $G$ and $H$, they are called isomorphic if there exists an isomorphism $\phi$ from $G$ to $H$, that is, $\phi: V(G) \longrightarrow V(H)$ is a bijection satisfying: $x \sim y$ in $G$ if and only if $\phi(x) \sim \phi(y)$ in $H$. An automorphism of $G$ is an isomorphism from $G$ to itself. The set $\operatorname{Aut}(G)$ of all automorphisms of $G$ forms a group under the composition of the transformations. $G$ is said to be vertex transitive if, for any $x, y \in V(G)$, there exists $\phi \in \operatorname{Aut}(G)$ such that $\phi(x)=y$. A subset $W \subseteq V(G)$ is called a fixing set (or determining set) of $G$ if the only automorphism of $G$ that fixes every vertex in $W$ is the identity, and the fixing number (or determining number) of $G$, denoted by fix $(G)$, is the smallest size of such a set. For $x, y \in V(G)$, the distance between them, written as $d(x, y)$, is the length of the shortest path joining them, and the diameter of $G$ is the largest distance between pairs of vertices of $G$. If $W=\left\{w_{1}, w_{2}, \ldots, w_{k}\right\}$ is an ordered subset of $V(G)$, then the $k$-dimensional vector $\left(d\left(x, w_{1}\right), \ldots, d\right.$ $\left.\left(x, w_{k}\right)\right)$ is called the representation of $x$ with respect to $W$, and $x$ is said to be resolved by $W$ if 
$\left(d\left(x, w_{1}\right), \ldots, d\left(x, w_{k}\right)\right) \neq\left(d\left(y, w_{1}\right), \ldots, d\left(y, w_{k}\right)\right)$ for any other vertex $y \in V(G)$. Moreover, $W$ is called a resolving set (or locating set) of $G$ if distinct vertices of $G$ have distinct representations with respect to $W$, and the metric dimension (or locating number) of $G$, denoted by $\operatorname{dim}(G)$, is the minimum cardinality of such a set. A graph $G$ is called an FED-graph if $\operatorname{fix}(G)=\operatorname{dim}(G)$. All other unexplained notations and definitions on graph theory are standard (see [22] or [23] for details).

Here, we list some results on graphs, which will be used in this paper.

Proposition 1 (Handshaking lemma, [22], Theorem 1.1). The number of edges in a graph $G$ is $(1 / 2) \sum_{x \in V(G)} \operatorname{deg}(x)$.

Proposition 2 (see [22], p. 18). A graph as well as its complement has the same automorphisms.

Proposition 3 (see [11], Theorem 1). A finite graph and its complement are of the same fixing number.

Proposition 4 (see [24], Lemma 2.8). Let $G$ be a connected graph. If $G$ has no singleton, then it is an FED-graph, and $\operatorname{fix}(G)=\operatorname{dim}(G)=|V(G)|-t w(G)$.

Proposition 5 (see [22], Theorem 3.5). A connected graph is Eulerian if and only if each vertex is of even degree.

Proposition 6 (Kuratowski's theorem, [22], Theorem 10.30). A graph is planar if and only if it contains no subdivisions of $K_{5}$ or $K_{3,3}$, where $K_{n, n}$ stands for the balanced complete bipartite graph of order $2 n$.

Proposition 7 (see [22], Corollary 18.12). If G is a connected graph in which all vertices are of odd degree, then it is Hamiltonian.

Proposition 8 (Chvátal-Erdős theorem, [22], Theorem 18.10). Let $G$ be a connected graph on at least 3 vertices with independent number $\iota$ and connectivity $\kappa$. If $\iota \leq \kappa$, then $G$ is Hamiltonian.

Proposition 9 (see [25]). Let $G$ be a 2-connected graph with minimum degree $\delta$ and independent number $\iota$. If $\delta \geq \max \{(|V(G)|+2 / 3), \iota\}$, then $G$ is Hamiltonian.

\section{Zero Component Graph of a Vector Space}

We first show that the zero component graph of $\mathbb{V}$ does not depend on the choice of the bases.

Theorem 1. Let $A=\left\{\varepsilon_{1}, \ldots, \varepsilon_{n}\right\}$ and $B=\left\{\eta_{1}, \ldots, \eta_{n}\right\}$ be bases of $\mathbb{V}$, then $\Gamma(\mathbb{V}, A)$ and $\Gamma(\mathbb{V}, B)$ are isomorphic.

Proof. Clearly, there exists an invertible linear transformation $f$ on $\mathbb{V}$ such that $f\left(\varepsilon_{i}\right)=\eta_{i}$ for any $1 \leq i \leq n$. Considering the restriction of $f$ on $\mathbb{V}_{*}(A)$, we see that $f$ is also a bijection from $\mathbb{V}_{*}(A)$ to $\mathbb{V}_{*}(B)$. For any $\alpha, \alpha^{\prime} \in \mathbb{V}_{*}(A)$, we obtain that $\alpha \sim \alpha^{\prime}$ in $\Gamma(\mathbb{V}, A)$ if and only if there exists some $1 \leq j \leq n$ such that $\varepsilon_{j} \in \mathscr{Z}_{A}(\alpha) \cap \mathscr{Z}_{A}\left(\alpha^{\prime}\right)$, if and only if $\eta_{j} \in \mathscr{Z}_{B}(f(\alpha)) \cap \mathscr{Z}_{B}\left(f\left(\alpha^{\prime}\right)\right)$, or equivalently $f(\alpha) \sim f\left(\alpha^{\prime}\right)$ in $\Gamma(\mathbb{V}, B)$. Therefore, $f$ is an isomorphism from $\Gamma(\mathbb{V}, A)$ to $\Gamma(\mathbb{V}, B)$.

Remark 1. In case the zero component graph of $\mathbb{V}$ does not depend on the choice of the bases, two vectors may be adjacent with respect to one basis but not adjacent with respect to another basis. For example, suppose that $\mathbb{V}$ is a 3 dimensional vector space over $F$, and $A=\left\{\varepsilon_{1}, \varepsilon_{2}, \varepsilon_{3}\right\}$ is a basis of $\mathbb{V}$. Then, $B=\left\{\varepsilon_{1}, \varepsilon_{1}+\varepsilon_{2}, \varepsilon_{2}+\varepsilon_{3}\right\}$ is also a basis of $\mathbb{V}$. It is easily seen that $\left(\varepsilon_{1}+\varepsilon_{2}\right) \times\left(\varepsilon_{2}+\varepsilon_{3}\right)$ in $\Gamma(\mathbb{V}, A)$, but $\left(\varepsilon_{1}+\right.$ $\left.\varepsilon_{2}\right) \sim\left(\varepsilon_{2}+\varepsilon_{3}\right)$ in $\Gamma(\mathbb{V}, B)$.

As usual, denote by $e_{i} \in \mathbb{V}, 1 \leq i \leq n$, the vector with the $i$-th component 1 and the others 0 . It is well known that $E=$ $\left\{e_{1}, \ldots, e_{n}\right\}$ constructs a basis of $\mathbb{V}$. Theorem 1 shows that we need only to consider the zero component graph $\Gamma(\mathbb{V}, E)$ of $\mathbb{V}$. For the convenience of writing, we set $e=\sum_{i=1}^{n} e_{i}$ and use $\Gamma(\mathbb{V}), \mathbb{V}_{*}, \quad \mathcal{S}(\alpha), \mathscr{Z}(\alpha)$ for $\alpha \in \mathbb{V}$, as well as $\mathscr{Z}_{j}$ for $0 \leq j \leq n-1$, instead of $\Gamma(\mathbb{V}, E), \mathbb{V}_{*}(E), \mathcal{S}_{E}(\alpha), \mathscr{Z}_{E}(\alpha)$, as well as $\mathscr{Z}_{j}(E)$, respectively. Then, by Theorem 1 , we can restate the definition of the zero component graph as follows.

Definition 1. The zero component graph $\Gamma(\mathbb{V})$ of $\mathbb{V}$ is a graph defined as follows: $\Gamma(\mathbb{V})$ has $\mathbb{V}_{*}=\mathbb{V}^{*} \backslash \mathscr{Z}_{0}$ as its vertex set, and for distinct $\alpha, \beta \in \mathbb{V}_{*}, \alpha \sim \beta$ if and only if $\mathscr{Z}(\alpha) \cap \mathscr{E}(\beta) \neq \varnothing$.

Next, we describe the connectedness and diameter of $\Gamma(\mathbb{V})$.

\section{Lemma 1}

(i) If $n=2$, then $\Gamma(\mathbb{V})$ is a union of two complete graphs

(ii) If $n \geq 3$, then $\Gamma(\mathbb{V})$ is a connected graph with diameter 2

Proof. The result is clear for $n=2$. Let $n \geq 3$. Then, by $\left(e-e_{1}\right) \nsim\left(e-e_{2}\right)$, we see that $\Gamma(\mathbb{V})$ is not complete. On the other hand, for any distinct $\alpha, \beta \in \mathbb{V}_{*}$, if $\alpha+\beta$, then there exist $1 \leq k, l \leq n$ such that $e_{k} \in \mathscr{Z}(\alpha) \backslash \mathscr{Z}(\beta)$ and $e_{l} \in \mathscr{Z}(\beta) \backslash \mathscr{Z}(\alpha)$, and further $\alpha \sim\left(e-e_{k}-e_{l}\right) \sim \beta$. Consequently, $\Gamma(\mathbb{V})$ is a connected graph with diameter 2 .

\section{Zero Component Graph and Graph Isomorphism Problem}

In this section, we investigate the interrelationship between the isomorphism of two vector spaces and that of their zero component graphs. Before that, we study the twins and the number of twin sets of the zero component graph.

Lemma 2. Two vectors $\alpha, \beta \in \mathbb{V}_{*}$ are twins in $\Gamma(\mathbb{V})$ if and only if $\mathscr{Z}(\alpha)=\mathscr{Z}(\beta)$ (or equivalently $\mathcal{S}(\alpha)=\mathcal{S}(\beta)$ ).

Proof. The sufficiency is clear; thus, we need only to prove the necessity. Let $\alpha, \beta \in \mathbb{V}_{*}$ be twins in $\Gamma(\mathbb{V})$. If $\mathscr{Z}(\alpha) \neq \mathscr{Z}(\beta)$, then $\mathscr{Z}(\alpha) \nsubseteq \mathscr{Z}(\beta)$ or $\mathscr{Z}(\alpha) \nsupseteq \mathscr{Z}(\beta)$. Without 
loss of the generalization, assume $\mathscr{Z}(\alpha) \nsubseteq \mathscr{Z}(\beta)$; say $e_{j} \in \mathscr{Z}(\alpha) \backslash \mathscr{Z}(\beta)$ for some $1 \leq j \leq n$. It follows $\alpha \sim\left(e-e_{j}\right)$ and $\beta+\left(e-e_{j}\right)$, a contradiction. Thus, $\mathscr{Z}(\alpha) \subseteq \mathscr{Z}(\beta)$. Similarly, $\mathscr{Z}(\alpha) \supseteq \mathscr{Z}(\beta)$. Therefore, $\mathscr{Z}(\alpha)=\mathscr{Z}(\beta)$.

Lemma 3. Let $n \geq 3$. Then,

(i) The number of twin sets of $\Gamma(\mathbb{V})$ is $t w(\Gamma(\mathbb{V}))=2^{n}-2$

(ii) Any vertex in $\Gamma(\mathbb{V})$ is a singleton when $|F|=2$, but not a singleton when $|F| \neq 2$

Proof. (i) follows from $\mathbb{V}_{*}=\cup_{1 \leq i \leq n-1} \mathscr{Z}_{i}$ and the fact that $\mathscr{Z}_{i}$ is a union of $\left(\begin{array}{c}n \\ i\end{array}\right)$ twin sets in $\Gamma(\mathbb{V})$ (see Lemma 2), where $\left(\begin{array}{c}n \\ i\end{array}\right)=n ! / i !(n-i) !$

For (ii), we see that the case of $|F|=2$ is clear according to Lemma 2. Assume $|F| \neq 2$. For any $\alpha \in \mathbb{V}_{*}$ and any $x \in F \backslash\{0,1\}$, it is obvious that $\alpha$ and $x \alpha$ are twins. This shows that $\alpha$ is not a singleton in $\Gamma(\mathbb{V})$, which confirms (ii). (i).

The following result is a direct consequence of Lemma 1

Theorem 2. Let $n, m \geq 3$; let $\mathbb{V}$ and $\mathbb{W}$ be, respectively, an $n$-dimensional vector space and an m-dimensional vector space over $F$. Then, $\mathbb{V}$ is isomorphic to $\mathbb{W}$ as vector spaces if and only if $\Gamma(\mathbb{V})$ is isomorphic to $\Gamma(\mathbb{W})$.

In the following, we will prove that two vector spaces over distinct finite fields are isomorphic if and only if their zero component graphs are isomorphic. To this end, we give the vertex degree of $\Gamma(\mathbb{V})$.

Lemma 4. Let $n \geq 3, q=|F|$, and $\alpha \in \mathscr{Z}_{j}(1 \leq j \leq n-1)$. Then, the degree of $\alpha$ in $\Gamma(\mathbb{V})$ is $\operatorname{deg}(\alpha)=q^{n}-q^{n-j}(q-1)^{j}-2$.

Proof. Assume $\beta=\sum_{i=1}^{n-j} x_{i} e_{i} \in \mathscr{Z}_{j}$ with $x_{i} \in F^{*}$ and denote

$$
N_{i}(\beta)=\{\gamma \in \mathbb{V}|| \mathscr{Z}(\gamma) \cap \mathscr{Z}(\beta) \mid=i\}, \quad 1 \leq i \leq j .
$$

Clearly, any pair of $N_{1}(\beta), \ldots, N_{j}(\beta)$ do not intersect with each other, and $\left|N_{i}(\beta)\right|=\left(\begin{array}{c}j \\ i\end{array}\right) q^{n-j}(q-1)^{j-i}$. Then, by $N(\beta)=\left(\cup_{i=1}^{j} N_{i}(\beta)\right) \backslash\{0, \beta\}$, we get

$$
\begin{aligned}
\operatorname{deg}(\beta) & =\sum_{i=1}^{j}\left|N_{i}(\beta)\right|-2=\sum_{i=1}^{j}\left(\begin{array}{l}
j \\
i
\end{array}\right) q^{n-j}(q-1)^{j-i}-2 \\
& =q^{n}-q^{n-j}(q-1)^{j}-2 .
\end{aligned}
$$

For any $\alpha \in \mathscr{Z}_{j}$, we write $\alpha=\sum_{i=1}^{n-j} a_{i} e_{k_{i}}$ with $a_{i} \in F^{*}$ and $1 \leq k_{1}<\cdots<k_{n-j} \leq n$. Take a permutation $\sigma$ on $[n]$ such that $\sigma(i)=k_{i}$ for $1 \leq i \leq j$, and define the map $\phi_{\sigma}: \mathbb{V} \longrightarrow \mathbb{V}$ by $\phi_{\sigma}\left(\sum_{i=1}^{n} y_{i} e_{i}\right)=\sum_{i=1}^{n} y_{i} e_{\sigma(i)}$ for any $y_{i} \in F$. Then, the restriction of $\phi_{\sigma}$ on $\mathbb{V}_{*}$ is an automorphism of $\Gamma(\mathbb{V})$, and $\phi_{\sigma}^{-1}(\alpha)=\sum_{i=1}^{n-j} a_{i} e_{i} \in \operatorname{Tw}(\beta)$. Therefore, $\operatorname{deg}(\alpha)=\operatorname{deg}\left(\phi_{\sigma}^{-1}\right.$ $(\alpha))=\operatorname{deg}(\beta)=q^{n}-q^{n-j}(q-1)^{j}-2$.
By Lemma 4, we get the following results immediately.

Corollary 1. Let $n \geq 3$, and $q=|F|$. Then, the order and size $m$ of $\Gamma(\mathbb{V})$ are $q^{n}-(q-1)^{n}-1 \quad$ and $\left(\left(q^{n}-1\right)\left(q^{n}-2\right)-(q-1)^{n}\left[(q+1)^{n}-3\right] / 2\right)$, respectively.

Proof. It is easily seen that the order of $\Gamma(\mathbb{V})$ is $\left|\mathbb{V}_{*}\right|=|\mathbb{V}|-\left|\mathscr{Z}_{0}\right|-1=q^{n}-(q-1)^{n}-1$. For the size of $\Gamma(\mathbb{V})$, by Handshaking lemma (see Proposition 1), Lemma 4, and $\left|\mathscr{Z}_{j}\right|=\left(\begin{array}{c}n \\ j\end{array}\right)(q-1)^{n-j}$ for $1 \leq j \leq n-1$, we have

$$
\begin{aligned}
2 m & =\sum_{j=1}^{n-1}\left|\mathscr{E}_{j}\right|\left[q^{n}-q^{n-j}(q-1)^{j}-2\right], \\
& =\sum_{j=1}^{n-1}\left(\begin{array}{c}
n \\
j
\end{array}\right)(q-1)^{n-j}\left[q^{n}-q^{n-j}(q-1)^{j}-2\right], \\
& =\left(q^{n}-1\right)\left(q^{n}-2\right)-(q-1)^{n}\left[(q+1)^{n}-3\right],
\end{aligned}
$$

from which it follows the result.

Corollary 2. Let $n \geq 3$, and $q=|F|$. Then, the maximum and minimum degree of $\Gamma(\mathbb{V})$ are $q^{n}-q(q-1)^{n-1}-2$ and $q^{n-1}-2$, respectively.

Corollary 3. Let $n \geq 3$, and let $F$ be a finite field. Then, $\Gamma(\mathbb{V})$ is Eulerian if and only if $|F|$ is even.

Proof. When $|F|$ is even (resp., odd), by Lemma 4, we obtain that each vertex in $\Gamma(\mathbb{V})$ is of even degree (resp., odd degree), which shows $\Gamma(\mathbb{V})$ is (resp., not) Eulerian.

Finally, we show that two vector spaces over distinct finite fields are isomorphic if and only if their zero component graphs are isomorphic.

Theorem 3. Let $n, m \geq 2 ; \mathbb{V}$ an $n$-dimensional vector space over a field with $q_{1}$ elements; and $\mathbb{W}$ an $m$-dimensional vector space over a field with $q_{2}$ elements. Then, $\mathbb{V}$ is isomorphic to $\mathbb{W}$ as vector spaces if and only if $\Gamma(\mathbb{V})$ is isomorphic to $\Gamma(\mathbb{W})$.

Proof. The necessity is obvious; thus, it suffices to prove the sufficiency. Assume that $\Gamma(\mathbb{V})$ is isomorphic to $\Gamma(\mathbb{W})$. If $n=2$, then by Lemma 1 (i), we know that $\Gamma(\mathbb{V})$ is a union of two complete graphs each of which is of order $q_{1}-1$, and so is $\Gamma(\mathbb{W})$. Again, by Lemma 1 (i), we have $m=2$ and $q_{1}=q_{2}$. Similarly, one may get $n=2$ and $q_{1}=q_{2}$ when $m=2$. Hence, $\mathbb{V}$ and $\mathbb{W}$ are isomorphic as vector spaces when $n=2$ or $m=2$. Now, let $n, m \geq 3$. Lemma 3 (i) shows that the numbers of twin sets of $\Gamma(\mathbb{V})$ and $\Gamma(\mathbb{W})$ are $2^{n}-2$ and $2^{m}-2$, respectively, which follows $n=m$. On the other hand, the minimum degrees of $\Gamma(\mathbb{V})$ and $\Gamma(\mathbb{W})$ are, respectively, $q_{1}^{n-1}-2$ and $q_{2}^{m-1}-2$ according to Corollary 2 . Then, by $n=m$, we obtain $q_{1}=q_{2}$. Thus, $\mathbb{V}$ and $\mathbb{W}$ are isomorphic as vector spaces. 


\section{Automorphism of $\Gamma(\mathbb{V})$}

We first construct two types of standard automorphisms of $\Gamma(\mathbb{V})$ as follows.

Definition 2. Let $G$ be a graph and $\rho$ a bijection on $V(G)$. If $\rho$ permutates the twins in $G$, then it is called a regular automorphism of $G$.

Definition 3. Denote by $\operatorname{Sym}(n)$ the symmetric group consisting of all permutations on [ $n$ ]. If $\sigma \in \operatorname{Sym}(n)$, then the map $\phi_{\sigma}: \mathbb{V} \longrightarrow \mathbb{V}, \sum_{i=1}^{n} a_{i} e_{i} \mapsto \sum_{i=1}^{n} a_{i} e_{\sigma(i)}$ is an invertible linear transformation on $\mathbb{V}$. By the proof of Theorem 1, we know that the restriction of $\phi_{\sigma}$ on $\mathbb{V}_{*}$ is an automorphism of $\Gamma(\mathbb{V})$, called a permutation automorphism of $\Gamma(\mathbb{V})$.

\section{Lemma 5}

(i) Let $\rho$ and $\phi_{\sigma}$ be defined as above. Then, $\phi_{\sigma}^{-1} \cdot \rho \cdot \phi_{\sigma}$ is a regular automorphism of $\Gamma(\mathbb{V})$.

(ii) If an automorphism $\phi$ of $\Gamma(\mathbb{V})$ is a regular automorphism but also a permutation automorphism, then $\phi$ is the identity.

Proof. For any $\alpha=\sum_{i=1}^{n} a_{i} e_{i} \in \mathbb{V}_{*}$ with $a_{i} \in F$, it is clear that $\rho \phi_{\sigma}(\alpha)=\rho\left(\sum_{i=1}^{n} a_{i} e_{\sigma(i)}\right)$ and $\sum_{i=1}^{n} a_{i} e_{\sigma(i)}$ are twins. By Lemma 2, we assume $\rho\left(\sum_{i=1}^{n} a_{i} e_{\sigma(i)}\right)=\sum_{i=1}^{n} a_{i} b_{i} e_{\sigma(i)}$ with $b_{i} \in F^{*}$. Then,

$$
\phi_{\sigma}^{-1} \rho \phi_{\sigma}(\alpha)=\phi_{\sigma}^{-1}\left(\sum_{i=1}^{n} a_{i} b_{i} e_{\sigma(i)}\right)=\sum_{i=1}^{n} a_{i} b_{i} e_{i} .
$$

Again, by Lemma 2 , we know that $\phi_{\sigma}^{-1} \rho \phi_{\sigma}(\alpha)$ and $\alpha$ are twins. By the arbitrariness of $\alpha$, we prove (i).

Assume $\phi=\rho=\phi_{\sigma}$ with $\sigma \in \operatorname{Sym}(n)$. For $1 \leq i \leq n$, by applying $\phi$ on $e_{i}$, we have $\rho\left(e_{i}\right)=e_{\sigma(i)}$, which shows that $e_{\sigma(i)}$ and $e_{i}$ are twins. It follows $\sigma(i)=i$ for any $1 \leq i \leq n$. Hence, $\sigma=1$, and further $\phi_{\sigma}=1$ or $\phi=1$. This confirms (ii).

Next, we need to present a result on the zero-divisor graph. Let $R$ be a commutative ring, then the zero-divisor graph $\Gamma_{z}(R)$ of $R$ is a graph defined as follows: $\Gamma_{z}(R)$ has vertex set consisting of all nonzero zero-divisors of $R$, and two nonzero zerodivisors are adjacent in $\Gamma_{z}(R)$ if and only if their product in $R$ is 0 . If $|F|=2$, we denote by $\mathbb{Z}_{2}^{n}$ the ring which has $\mathbb{V}$ as an additive group and the multiplication of two elements $\alpha=\sum_{i=1}^{n}$ $a_{i} e_{i}, \beta=\sum_{i=1}^{n} b_{i} e_{i} \in \mathbb{V}$ with $a_{i}, b_{i} \in F$ are defined naturally:

$$
\alpha \cdot \beta=\sum_{i=1}^{n} a_{i} b_{i} e_{i}
$$

It is clear that $\mathbb{V}_{*}$ is also the vertex set of $\Gamma_{z}\left(\mathbb{Z}_{2}^{n}\right)$. In [24], the automorphisms of $\Gamma_{z}\left(\mathbb{Z}_{2}^{n}\right)$ were described as follows.

Proposition 10 (see [24], Theorem 1). Any automorphism of $\Gamma_{z}\left(\mathbb{Z}_{2}^{n}\right)$ is a permutation automorphism, and $\operatorname{Aut}\left(\Gamma_{z}\left(\mathbb{Z}_{2}^{n}\right)\right) \cong$ $\operatorname{Sym}(n)$.

The following result shows that the relation between $\Gamma(\mathbb{V})$, for $|F|=2$, and $\Gamma_{z}\left(\mathbb{Z}_{2}^{n}\right)$.
Lemma 6. If $|F|=2$, then the complement graph $\overline{\Gamma(\mathbb{V})}$ of $\Gamma(\mathbb{V})$ is isomorphic to $\Gamma_{z}\left(\mathbb{Z}_{2}^{n}\right)$.

Proof. Define $g: \mathbb{V}_{*} \longrightarrow \mathbb{V}_{*}$ by $g(\alpha)=e-\alpha, \forall \alpha \in \mathbb{V}_{*}$. It is clear that $g$ is a bijection on $\mathbb{V}_{*}$. Moreover, for any $\alpha, \beta \in \mathbb{V}_{*}$, we see that $\alpha \star \beta$ in $\Gamma(\mathbb{V})$ (or equivalently $\mathscr{Z}(\alpha) \cap \mathscr{Z}(\beta)=\varnothing$ ) if and only if $\mathcal{S}(g(\alpha)) \cap \mathcal{S}(g(\beta))=\varnothing$, if and only if $g(\alpha) g(\beta)=0$. Thus, $\alpha \sim \beta$ in $\overline{\Gamma(\mathbb{V})}$ if and only if $g(\alpha) \sim g(\beta)$ in $\Gamma_{z}\left(\mathbb{Z}_{2}^{n}\right)$, and therefore, $g$ is an isomorphism from $\overline{\Gamma(\mathbb{V})}$ to $\Gamma_{z}\left(\mathbb{Z}_{2}^{n}\right)$

By Proposition 2, Proposition 10, and Lemma 6, we can immediately determine the automorphisms of $\Gamma(\mathbb{V})$ for $|F|=2$.

Lemma 7. If $|F|=2$, then any automorphism of $\Gamma(\mathbb{V})$ is a permutation automorphism, and $A u t(\Gamma(\mathbb{V})) \cong \operatorname{Sym}(n)$.

In the following, we consider the case for an arbitrary finite-dimensional vector space. Before that, we introduce the quotient graph $\widetilde{\Gamma(\mathbb{V})}$ of $\Gamma(\mathbb{V})$, which is defined as follows: $\widetilde{\Gamma(\mathbb{V})}$ has all twin sets of $\Gamma(\mathbb{V})$ as its vertex set, and for any $\alpha, \beta \in \mathbb{V}_{*}, \quad \operatorname{Tw}(\alpha) \sim \operatorname{Tw}(\beta)$ in $\widetilde{\Gamma(\mathbb{V})}$ if and only if $\operatorname{Tw}(\alpha) \neq \operatorname{Tw}(\beta)$ as well as $\mathscr{Z}(\alpha) \cap \mathscr{Z}(\beta) \neq \varnothing$. It is easily seen that the definition is well defined since $\mathscr{Z}(\alpha) \cap \mathscr{Z}(\beta) \neq \varnothing \Leftrightarrow \mathscr{Z}\left(\alpha^{\prime}\right) \cap \mathscr{Z}\left(\beta^{\prime}\right) \neq \varnothing$ for any $\alpha^{\prime} \in \operatorname{Tw}(\alpha)$ and $\beta^{\prime} \in \operatorname{Tw}(\beta)$ (see Lemma 2 ), and $\widetilde{\Gamma(\mathbb{V})}$ is isomorphic to $\Gamma\left(\mathbb{V}_{2}\right)$, where $\mathbb{V}_{2}$ is the vector space over the field $\mathbb{Z}_{2}$. Let $\phi$ be an automorphism of $\Gamma(\mathbb{V})$. We define $\tilde{\phi}$ on $\widetilde{\Gamma(\mathbb{V})}$ by

$$
\widetilde{\phi}(\operatorname{Tw}(\alpha))=\operatorname{Tw}(\phi(\alpha)), \quad \forall \alpha \in \mathbb{V}_{*} .
$$

Lemma 8. Let $\phi$ be an automorphism of $\Gamma(\mathbb{V})$; then, $\tilde{\phi} d e$ fined as in (8) is an automorphism of $\widehat{\Gamma(\mathbb{V})}$.

Proof. If $\operatorname{Tw}(\alpha)=\operatorname{Tw}(\beta)$ for $\alpha, \beta \in \mathbb{V}_{*}$, then $\alpha, \beta \in \mathbb{V}_{*}$ are twins in $\Gamma(\mathbb{V})$. Furthermore, $\phi(\alpha)$ and $\phi(\beta)$ are also twins in $\Gamma(\mathbb{V})$, following $\operatorname{Tw}(\phi(\alpha))=\operatorname{Tw}(\phi(\beta))$. Hence, $\widetilde{\phi}$ is well defined. It is clear that $\widetilde{\phi}$ is a bijection. Moreover, from the fact that $\phi(\alpha) \sim \phi(\beta)$ in $\Gamma(\mathbb{V})$ if and only if $\alpha \sim \beta$ in $\Gamma(\mathbb{V})$, it follows that $\operatorname{Tw}(\phi(\alpha)) \sim \operatorname{Tw}(\phi(\beta))$ in $\widetilde{\Gamma(\mathbb{V})}$ if and only if $\operatorname{Tw}(\alpha) \sim \operatorname{Tw}(\beta)$ in $\widetilde{\Gamma(\mathbb{V})}$. Thus, $\widetilde{\phi}$ is an automorphism of $\Gamma(\mathbb{V})$.

Now, we can consider the case for an arbitrary finitedimensional vector space.

Theorem 4. Let $n \geq 2$. Then, a bijection on $\mathbb{V}_{*}$ is an automorphism of $\Gamma(\mathbb{V})$ if and only if it can be uniquely decomposed into the product of a permutation automorphism and a regular automorphism of $\Gamma(\mathbb{V})$.

Proof. The uniqueness of decomposition is clear according to Lemma 5 (ii); thus, it suffices to prove the existence. When $|F|=2$, we get the result by Lemma 7. Now, assume $|F| \neq 2$.

For any automorphism $\phi$ of $\Gamma(\mathbb{V})$, define $\widetilde{\phi}$ as in (8). Then, by Lemma 8 , we know that $\tilde{\phi}$ is an automorphism of $\widetilde{\Gamma(\mathbb{V})}$, which is isomorphic to $\Gamma\left(\mathbb{V}_{2}\right)$ (where $\mathbb{V}_{2}$ is the vector space over the field $\mathbb{Z}_{2}$ ). Applying Lemma 7 , we see that there exists a permutation $\sigma \in \operatorname{Sym}(n)$ such that 


$$
\tilde{\phi}(\operatorname{Tw}(\alpha))=\operatorname{Tw}\left(\phi_{\sigma}(\alpha)\right), \quad \forall \alpha \in \mathbb{V}_{*} .
$$

By the definition of $\widetilde{\phi}$, we have $\operatorname{Tw}(\phi(\alpha))=\operatorname{Tw}\left(\phi_{\sigma}(\alpha)\right)$, or, equivalently, $\phi(\alpha)$ and $\phi_{\sigma}(\alpha)$ are twins. Consequently, there exists a regular automorphism $\rho$ of $\Gamma(\mathbb{V})$ such that $\phi(\alpha)=\rho\left(\phi_{\sigma}(\alpha)\right)$ for any $\alpha \in \mathbb{V}_{*}$, and therefore $\phi=\rho \cdot \phi_{\sigma}$.

The following is a direct consequence of Lemma 1 (i) and Theorem 4.

Corollary 4. $\Gamma(\mathbb{V})$ is vertex transitive if and only if $n=2$.

Denote by $\operatorname{Reg}(\Gamma(\mathbb{V}))$ and $\operatorname{Per}(\Gamma(\mathbb{V}))$ the sets consisting of all regular automorphisms and all permutation automorphisms of $\Gamma(\mathbb{V})$, respectively. Then, $\operatorname{Reg}(\Gamma(\mathbb{V}))$ and $\operatorname{Per}(\Gamma(\mathbb{V}))$ are subgroups of the automorphism group $\operatorname{Aut}(\Gamma(\mathbb{V}))$ of $\Gamma(\mathbb{V})$. Finally, by Theorem 4, we can describe $\operatorname{Aut}(\Gamma(\mathbb{V}))$ and its order as follows.

\section{Corollary 5}

(i) $\operatorname{Aut}(\Gamma(\mathbb{V}))=\operatorname{Per}(\Gamma(\mathbb{V})) \ltimes \operatorname{Reg}(\Gamma(\mathbb{V}))$. In particular, $\operatorname{Aut}(\Gamma(\mathbb{V}))=\operatorname{Per}(\Gamma(\mathbb{V}))$ when $|F|=2$.

(ii) If $q=|F|$, then $|A u t(\Gamma(V))|=n ! \prod_{i=1}^{n-1}\left((q-1)^{i} !\right)^{k}$ with $k=\left(\begin{array}{c}n \\ i\end{array}\right)$.

Proof. Theorem 4 shows $\operatorname{Aut}(\Gamma(\mathbb{V}))=\operatorname{Per}(\Gamma(\mathbb{V}))$. $\operatorname{Reg}(\Gamma(\mathbb{V}))$. Then, by $\operatorname{Per}(\Gamma(\mathbb{V})) \cap \operatorname{Reg}(\Gamma(\mathbb{V}))=1 \quad$ (see Lemma 5 (ii)) and the fact that $\operatorname{Reg}(\Gamma(\mathbb{V})$ ) is a normal subgroup of $\operatorname{Aut}(\Gamma(\mathbb{V}))$ (see Lemma 5 (i)), we obtain the result of (i).

(ii) follows from (i), $\operatorname{Per}(\Gamma(\mathbb{V})) \cong \operatorname{Sym}(n)$, and $\operatorname{Reg}(\Gamma(\mathbb{V})) \cong \prod_{i=1}^{n-1}\left(\prod_{j=1}^{k} \operatorname{Sym}\left((q-1)^{i}\right)\right)$.

\section{Fixing Number and Metric Dimension of $\Gamma(\mathbb{V})$}

In this section, we always assume $F$ is a finite field with $q(\geq 2)$ elements. If $n=2$, nothing needs to do for the metric dimension of $\Gamma(\mathbb{V})$ according to Lemma 2; the fixing number $\Gamma(\mathbb{V})$ is 1 when $q=2$, and $2(q-2)$ otherwise. In the following, we consider the case for $n \geq 3$.

Firstly, by Proposition 4, Lemma 3, and Corollary 1, we have the following result immediately.

Lemma 9. Let $n \geq 3$ and $q \geq 3$. Then, $\Gamma(\mathbb{V})$ is an FED-graph, and $\operatorname{fix}(\Gamma(\mathbb{V}))=\operatorname{dim}(\Gamma(\mathbb{V}))=q^{n}-(q-1)^{n}-2^{n}+1$.

Next, we give the value of the fixing number of $\Gamma(\mathbb{V})$ for $n \geq 3$ and $q=2$.

Lemma 10. Let $n \geq 3$ and $q=2$. Then, $f i x(\Gamma(\mathbb{V}))=\left\lceil\log _{2} n\right\rceil$.

Proof. By Proposition 3 and Lemma 6, we have $\operatorname{fix}(\Gamma(\mathbb{V}))=\operatorname{fix}\left(\Gamma_{z}\left(\mathbb{Z}_{2}^{n}\right)\right)$. Furthermore, from fix $\left(\Gamma_{z}\left(\mathbb{Z}_{2}^{n}\right)\right)=$ $\left\lceil\log _{2} n\right\rceil$ (see [24], Theorem 3.5), it follows that fix $(\Gamma(\mathbb{V}))=\left\lceil\log _{2} n\right\rceil$.
The vector $\alpha=\sum_{i=1}^{n} a_{i} e_{i} \in \mathbb{V}$ with $a_{i} \in F$ is said to be $\left(\delta_{1}(\alpha), \delta_{2}(\alpha), \ldots, \delta_{n}(\alpha)\right)$-type, where

$$
\delta_{j}(\alpha)= \begin{cases}1, & \text { if } a_{j} \neq 0, \\ 0, & \text { if } a_{j}=0,\end{cases}
$$

for $1 \leq j \leq n$. The following result is trivial.

Lemma 11. If $q=2$, then all vectors in $\mathbb{V}_{*}$ are of different types.

Now, we can calculate the metric dimension of $\Gamma(\mathbb{V})$ for $n \geq 3$ and $q=2$.

Lemma 12. Let $n \geq 3$ and $q=2$. Then, $\operatorname{dim}(\Gamma(\mathbb{V}))=n$ when $n \geq 4$, and $\operatorname{dim}(\Gamma(\mathbb{V}))=2$ when $n=3$.

Proof. When $n=3$, it is clear that $\operatorname{dim}(\Gamma(\mathbb{V})) \neq 1$ as shown in Figure 1.

Moreover, $W_{1}=\left\{e_{1}+e_{3}, e_{2}+e_{3}\right\}$ is a resolving set of $\Gamma(\mathbb{V})$. So, $\operatorname{dim}(\Gamma(\mathbb{V}))=2$. Now, let $n \geq 4$. We first assert that $W_{2}=\left\{e-e_{j} \mid 1 \leq j \leq n\right\} \subseteq \mathbb{V}_{*}$ is a resolving set of $\Gamma(\mathbb{V})$. For any $\alpha \in \mathbb{V}_{*}$, it is of $\left(\delta_{1}(\alpha), \ldots, \delta_{n}(\alpha)\right)$-type, and its presentation with respect to $W_{2}$ is $\left(\delta_{1}(\alpha)+1, \ldots, \delta_{n}(\alpha)+1\right)$, where $\delta_{j}(\alpha)$ is defined as in (10). Since the types of all vectors in $\mathbb{V}_{*}$ are different (see Lemma 11), the same is for their representation with respect to $W_{2}$. Hence, $W_{2}$ is a resolving set of $\Gamma(\mathbb{V})$, and therefore $\operatorname{dim}(\Gamma(\mathbb{V})) \leq n$. Next, we need to show $\operatorname{dim}(\Gamma(\mathbb{V})) \geq n$. Suppose $\operatorname{dim}(\Gamma(\mathbb{V}))=k$ and that $U=\left\{\alpha_{1}, \alpha_{2}, \ldots, \alpha_{k}\right\} \subseteq \mathbb{V}$ is a resolving set of $\Gamma(\mathbb{V})$. Define the $\operatorname{map} h: \mathbb{V}_{*} \backslash U \longrightarrow \mathbb{Z}_{2}^{k}$ as

$$
h(\beta)=\left(d\left(\beta, \alpha_{1}\right)-1, d\left(\beta, \alpha_{2}\right)-1, \ldots, d\left(\beta, \alpha_{k}\right)-1\right), \quad \forall \beta \in \mathbb{V}_{*} \backslash U .
$$

Lemma 1 (ii) shows that $h$ is well defined. Moreover, for any $\beta \neq \gamma \in \mathbb{V}_{*} \mid U$, since their representation with respect to $U$ is different, we see that $h(\beta) \neq h(\gamma)$. Consequently, $h$ is injective, and further $2^{n}-2-k=\left|\mathbb{V}_{*}\right| U|\leq| \mathbb{Z}_{2}^{k} \mid=2^{k}$. It follows that $k \geq n$. Thus, $\operatorname{dim}(\Gamma(\mathbb{V}))=n$.

Finally, combining Lemmas 9,10 , and 12 , we get the following theorem.

Theorem 5. Let $n \geq 3$ and $q=|F|$. Then, $\Gamma(\mathbb{V})$ is an FED-graph if and only if $q \geq 3$, or $n=3$ as well as $q=2$. Moreover, $f i x(\Gamma(\mathbb{V}))=\operatorname{dim}(\Gamma(\mathbb{V}))=q^{n}-(q-1)^{n}-2^{n}+1$ when $q \geq 3 ; f i x(\Gamma(\mathbb{V}))=\operatorname{dim}(\Gamma(\mathbb{V}))=2$ when $n=3, q=2$; and $f i x(\Gamma(\mathbb{V}))=\left\lceil\log _{2} n\right\rceil$ as well as $\operatorname{dim}(\Gamma(\mathbb{V}))=n$ when $n \geq 4, q=2$.

\section{Other Properties of $\Gamma(\mathbb{V})$}

We first consider the girth, minimum independent dominating sets, maximum independent sets, and planarity of $\Gamma(\mathbb{V})$ when $F$ is an arbitrary field.

Theorem 6. Each vertex in $\Gamma(\mathbb{V})$ belongs to some triangle in $\Gamma(\mathbb{V})$. Furthermore, the girth of $\Gamma(\mathbb{V})$ is 3 . 


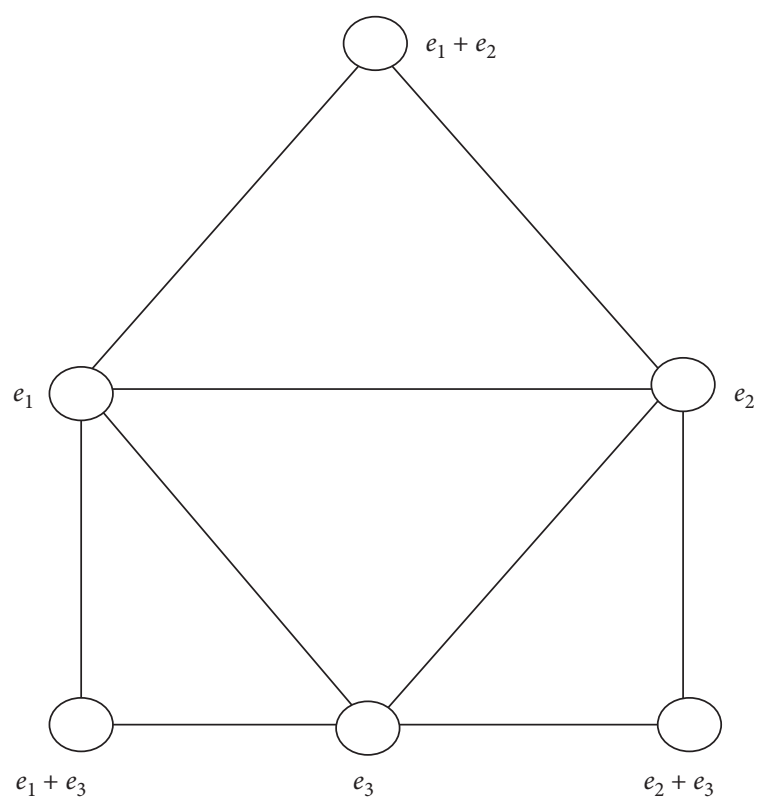

Figure 1: $\Gamma(\mathbb{V})$ for $n=3$ and $q=2$.

Proof. The case for $n=2$ is clear according to Lemma 1 (i). Now, let $n \geq 3$. For any $\alpha \in \mathbb{V}_{*}$, if $|\mathscr{Z}(\alpha)|=n-1$, assume $e_{k} \notin \mathscr{Z}(\alpha)$ for some $1 \leq k \leq n$. Choose distinct $i, j \in[n] \backslash\{k\}$; then $\alpha \sim e_{i} \sim e_{j} \sim \alpha$; if $|\mathscr{Z}(\alpha)| \leq n-2$, say $e_{s}, e_{t} \notin \mathscr{Z}(\alpha)$ for some $1 \leq s, t \leq n$, then $\alpha \sim e_{s} \sim e_{t} \sim \alpha$. Therefore, in any case, $\alpha$ belongs to a triangle in $\Gamma(\mathbb{V})$.

Lemma 13. Let $n \geq 3$. Then, the dominating number of $\Gamma(\mathbb{V})$ is 2 .

Proof. This is a direct consequence of the fact that any vertex is adjacent to at least one of $\left\{e_{1}, e_{2}\right\}$.

Theorem 7. Let $n \geq 3$. Then, $I=\{\alpha, \beta\} \subseteq \mathbb{V}_{*}$ is a minimum independent dominating set of $\Gamma(\mathbb{V})$ if and only if $\mathscr{Z}(\alpha) \cap \mathscr{Z}(\beta)=\varnothing$ as well as $\mathscr{Z}(\alpha) \cup \mathscr{Z}(\beta)=\left\{e_{1}, \ldots, e_{n}\right\}$.

Proof. Let $I=\{\alpha, \beta\} \subseteq \mathbb{V}_{*}$ be an independent dominating set of $\Gamma(\mathbb{V})$. It is clear that $\mathscr{Z}(\alpha) \cap \mathscr{Z}(\beta)=\varnothing$. If $\mathscr{Z}(\alpha) \cup \mathscr{Z}(\beta) \neq\left\{e_{1}, \ldots, e_{n}\right\}$, say $e_{j} \notin \mathscr{Z}(\alpha) \cup \mathscr{Z}(\beta)$ for some $1 \leq j \leq n$, then $\alpha \nsim\left(e-e_{j}\right)$ and $\beta \nsim\left(e-e_{j}\right)$. This implies that $I$ is not a dominating set of $\Gamma(\mathbb{V})$, a contradiction. Hence, $\mathscr{Z}(\alpha) \cup \mathscr{Z}(\beta)=\left\{e_{1}, \ldots, e_{n}\right\}$.

Conversely, assume $\mathscr{Z}(\alpha) \cap \mathscr{Z}(\beta)=\varnothing$ and $\mathscr{Z}(\alpha) \cup \mathscr{Z}$ $(\beta)=\left\{e_{1}, \ldots, e_{n}\right\}$ for $\alpha, \beta \in \mathbb{V}_{*}$. By $\mathscr{Z}(\alpha) \cap \mathscr{Z}(\beta)=\varnothing$, we see that $I=\{\alpha, \beta\}$ is an independent set of $\Gamma(\mathbb{V})$. On the other hand, for any $\gamma \in \mathbb{V}_{*}$, since $\mathscr{Z}(\alpha) \cap \mathscr{Z}(\gamma)=\varnothing$ implies $\mathscr{Z}(\beta) \cap \mathscr{Z}(\gamma) \neq \varnothing$, we obtain that $\alpha+\gamma$ leads to $\beta \sim \gamma$, which shows that $I$ is a dominating set. Therefore, $I$ is an independent dominating set, and further a minimum independent dominating set of $\Gamma(\mathbb{V})$.

Lemma 14. The independence number of $\Gamma(\mathbb{V})$ is $n$ (i.e., the dimension of $\mathbb{V}$ ).
Proof. Let $\iota$ be the independence number of $\Gamma(\mathbb{V})$. Clearly, $\left\{e-e_{i} \mid 1 \leq i \leq n\right\} \subseteq \mathbb{V}_{*}$ is an independent set of $\Gamma(\mathbb{V})$. This implies $\imath \geq n$. Now, it suffices to show $\iota \leq n$. Suppose to the contrary, $\iota \geq n+1$, and assume that $\left\{\alpha_{j} \mid 1 \leq j \leq n+1\right\}$ is an independent set of $\Gamma(\mathbb{V})$. Then, $\mathscr{Z}\left(\alpha_{1}\right), \ldots, \mathscr{Z}\left(\alpha_{n+1}\right)$ are disjoint nonempty sets. From $\cup_{j=1}^{n+1} \mathscr{Z}\left(\alpha_{j}\right) \subseteq\left\{e_{i} \mid 1 \leq i \leq n\right\}$, it follows that $n+1 \leq \sum_{j=1}^{n+1}\left|\mathscr{Z}\left(\alpha_{j}\right)\right| \leq\left|\cup_{j=1}^{n+1} \mathscr{E}\left(\alpha_{j}\right)\right| \leq n$, a contradiction.

Theorem 8. The set $I=\left\{\alpha_{j} \mid 1 \leq j \leq n\right\} \subseteq \mathbb{V}_{*}$ is a maximum independent set of $\Gamma(\mathbb{V})$ if and only if $I \subseteq \mathscr{Z}_{1}$, and $\mathscr{Z}\left(\alpha_{1}\right), \ldots, \mathscr{Z}\left(\alpha_{n}\right)$ are disjoint.

Proof. The sufficiency is obvious; thus, we need only to prove the necessity. Let $I=\left\{\alpha_{j} \mid 1 \leq j \leq n\right\} \subseteq \mathbb{V}_{*}$ be an independent set of $\Gamma(\mathbb{V})$. Clearly, $\mathscr{Z}\left(\alpha_{1}\right), \ldots, \mathscr{Z}\left(\alpha_{n}\right)$ are disjoint. It suffices to show $\alpha_{j} \in \mathscr{Z}_{1}$; that is, $\alpha_{j}$ has exactly one zero component, $1 \leq j \leq n$. The case for $n=2$ is clear. Let $n \geq 3$, and suppose, to the contrary, there exists some $1 \leq k \leq n$ such that $\alpha_{k} \notin \mathscr{Z}_{1}$. Without loss of the generalization, assume $e_{n-1}, e_{n} \in \mathscr{Z}\left(\alpha_{k}\right)$. For any $j \neq k$, by $\alpha_{j} \nsim \alpha_{k}$, we get $e_{n-1}, e_{n} \notin \mathscr{Z}\left(\alpha_{j}\right)$, and consequently $\cup_{j \neq k} \mathscr{Z}\left(\alpha_{j}\right) \subseteq\left\{e_{i} \mid 1 \leq\right.$ $i \leq n-2\}$. Hence, $\quad n-1 \leq \sum_{j \neq k}\left|\mathscr{E}\left(\alpha_{j}\right)\right| \leq\left|\cup_{j \neq k} \mathscr{X}\left(\alpha_{j}\right)\right|$ $\leq n-2$, a contradiction.

Theorem 9. $\Gamma(\mathbb{V})$ is planar if and only if $n=2$ and $|F| \leq 5$, or $n=3$ and $|F|=2$.

Proof. When $n=2$ (by Lemma 1 (i)), we know that $\Gamma(\mathbb{V})$ is a union of two complete graphs each of which is of order $|F|-1$. This follows that $\Gamma(\mathbb{V})$ is planar if and only if $|F| \leq 5$ (see Proposition 6). When $n=3$ and $|F|=2$, we obtain that $\Gamma(\mathbb{V})$ is planar as shown in Figure 1. When $n \geq 4$ (resp., $n=3$ and $|F| \geq 3)$, the subgraph of $\Gamma(\mathbb{V})$ induced by $\left\{e_{1}, e_{2}, e_{3}, e_{1}+e_{2}, e_{1}+e_{3}, e_{2}+e_{3}\right\} \quad$ (resp., $\left\{a e_{i} \mid a=1,2\right.$ and $\left.\left.1 \leq i \leq 3\right\}\right)$ is $K_{6}$ and further nonplanar.

Next, we provide a lower bound for the connectivity of $\Gamma(\mathbb{V})$ when $F$ is a finite field.

Lemma 15. Let $n \geq 3$ and $q=|F|$. Then, the connectivity of $\Gamma(\mathbb{V})$ is greater than or equal to $q^{n-2}-1$.

Proof. Let $\alpha$ and $\beta$ be two nonadjacent vertices in $\Gamma(\mathbb{V})$ and suppose that $X \subseteq \mathbb{V}_{*}$ such that $\alpha$ and $\beta$ are in different components of $\Gamma\left[\mathbb{V}_{*} \backslash X\right]$, the subgraph of $\Gamma(\mathbb{V})$ induced by $\mathbb{V}_{*} \backslash X$. It is easily seen that $X \supseteq(N(\alpha) \cap N(\beta))$; indeed, if $X \nsupseteq(N(\alpha) \cap N(\beta))$, then $\alpha \sim \gamma \sim \beta$ in $\Gamma\left[\mathbb{V}_{*} \backslash X\right]$ for any $\gamma \in(N(\alpha) \cap N(\beta)) \backslash X$, a contradiction. Assume $e_{k} \in \mathscr{Z}(\alpha)$, $e_{l} \in \mathscr{Z}(\beta)$ and set

$$
Y=\left\{\gamma \in \mathbb{V}_{*} \mid e_{k}, e_{l} \in \mathscr{Z}(\gamma)\right\} .
$$

From $\alpha \nsim \beta$, it follows that $e_{l} \notin \mathscr{Z}(\alpha)$ and $e_{k} \notin \mathscr{Z}(\beta)$, which implies $\alpha, \beta \notin Y$. Furthermore, $Y \subseteq(N(\alpha) \cap N(\beta)) \subseteq X$, and thus $|X| \geq|Y|=q^{n-2}-1$, from which we obtain the result.

Finally, we can characterize the hamiltonicity of $\Gamma(\mathbb{V})$ when $F$ is a finite field. 
Theorem 10. Let $n \geq 3$ and let $F$ be a finite field. Then, $\Gamma(\mathbb{V})$ is Hamiltonian.

Proof. If $|F|$ is odd, then each vertex in $\Gamma(\mathbb{V})$ is of odd degree (see Lemma 4). By Proposition 7 , we know that $\Gamma(\mathbb{V})$ is Hamiltonian. Now, let $|F|$ be even. If $n \geq 5$ or $|F| \geq 4$, we have $|F|^{n-2}-1 \geq n$; that is, the connectivity of $\Gamma(\mathbb{V})$ is greater than or equal to the independent number (see Lemma 14 and Lemma 15); then, by Chvátal-Erdős theorem (see Proposition 8), we obtain that $\Gamma(\mathbb{V})$ is Hamiltonian. The case for $n=3$ and $|F|=2$ is clear according to Figure 1. If $n=4$ and $|F|=2$, then $\Gamma(\mathbb{V})$ is 2 -connected with minimum degree $\delta=$ $2^{n-1}-2$ (see Corollary 2) and independent number $n$, which follows $\delta \geq \max \left\{\left(\left|\mathbb{V}_{*}\right|+2 / 3\right), n\right\}$. Applying a theorem of Nash-Williams (see Proposition 9), we see that $\Gamma(\mathbb{V})$ is Hamiltonian.

\section{Conclusion}

In this paper, we present an undirected simple graph, called the zero component graph $\Gamma(\mathbb{V})$, over a finite-dimensional vector space $\mathbb{V}$, and investigate various interrelationships among $\Gamma(\mathbb{V})$ and $\mathbb{V}$. The aim of this paper is to determine the automorphisms, compute the fixing number as well as metric dimension, and study other properties such as girth, minimum independent dominating sets, maximum independent sets, planarity, and hamiltonicity of $\Gamma(\mathbb{V})$. In addition, a lower bound for the connectivity is given. As a topic of further research, one can give the exact value of the connectivity and clique number of $\Gamma(\mathbb{V})$.

\section{Data Availability}

No data were used to support this study.

\section{Conflicts of Interest}

The authors declare no conflicts of interest.

\section{Acknowledgments}

This work was supported by the National Natural Science Foundation of China (no. 11661040).

\section{References}

[1] A. Das, "Nonzero component graph of a finite dimensional vector space," Communications in Algebra, vol. 44, no. 9, pp. 3918-3926, 2016.

[2] A. Das, "Subspace inclusion graph of a vector space," Communications in Algebra, vol. 44, no. 11, pp. 4724-4731, 2016.

[3] Z. Gu and Z.-X. Wan, "Orthogonal graphs of odd characteristic and their automorphisms," Finite Fields and Their Applications, vol. 14, no. 2, pp. 291-313, 2008.

[4] Z. Tang and Z.-X. Wan, "Symplectic graphs and their automorphisms," European Journal of Combinatorics, vol. 27, no. 1, pp. 38-50, 2006.

[5] A. Das, "On nonzero component graph of vector spaces over finite fields," Journal of Algebra and Its Applications, vol. 16, no. $1,2017$.
[6] M. Murtaza, I. Javaid, and M. Fazil, "Locating-dominating sets and identifying codes of a graph associated to a finite vector space," 2017.

[7] A. Das, "Non-zero component union graph of a finite-dimensional vector space," Linear and Multilinear Algebra, vol. 65, no. 6, pp. 1276-1287, 2017.

[8] X. Wang and D. Wong, "Automorphism group of the subspace inclusion graph of a vector space," Bulletin of the Malaysian Mathematical Sciences Society, vol. 42, no. 5, pp. 2213-2224, 2019.

[9] D. Erwin and F. Harary, "Destroying automorphisms by fixing nodes," Discrete Mathematics, vol. 306, no. 24, pp. 3244-3252, 2006.

[10] R. F. Bailey and P. J. Cameron, "Base size, metric dimension and other invariants of groups and graphs," Bulletin of the London Mathematical Society, vol. 43, pp. 209-242, 2011.

[11] M. Fazil, "Determining sets and related parameters in graphs," 2009.

[12] H. Benish, I. Javaid, and M. Murtaza, "Automorphism related parameters of a graph associated to a finite vector space," Utilitas Mathematica, vol. 111, pp. 35-48, 2019.

[13] U. Ali, S. A. Bokhary, K. Wahid, and G. Abbas, "On resolvability of a graph associated to a finite vector space," Journal of Algebra and its Applications, vol. 18, no. 2, 2019.

[14] D. Boutin, "Identifying graph automorphisms using determining sets," Electronic Journal of Combinatorics, vol. 13, 2006.

[15] R. F. Bailey, J. Cáceres, D. Garijo et al., "Resolving sets for Johnson and kneser graphs," European Journal of Combinatorics, vol. 34, no. 4, pp. 736-751, 2013.

[16] J. Cáceres, C. Hernando, M. Mora et al., "On the metric dimension of cartesian products of graphs," SIAM Journal on Discrete Mathematics, vol. 21, no. 2, pp. 423-441, 2007.

[17] M. Feng, X. Ma, and K. Wang, "The structure and metric dimension of the power graph of a finite group," European Journal of Combinatorics, vol. 43, pp. 82-97, 2015.

[18] S. Imran, M. Siddiqui, M. Imran, and M. Hussain, "On metric dimensions of symmetric graphs obtained by rooted product," Mathematics, vol. 6, no. 10, p. 191, 2018.

[19] J.-B. Liu, Z. Zahid, R. Nasir, and W. Nazeer, "Edge version of metric dimension and doubly resolving sets of the necklace graph," Mathematics, vol. 6, no. 11, p. 243, 2018.

[20] H. Raza, S. Hayat, M. Imran, and X.-F. Pan, "Fault-tolerant resolvability and extremal structures of graphs," Mathematics, vol. 7, no. 1, p. 78, 2019.

[21] J. Wang, L. Miao, and Y. Liu, "Characterization of $n$ n-vertex graphs of metric dimension $n-3$ by metric matrix," Mathematics, vol. 7, p. 497, 2019.

[22] J. A. Bondy and U. S. R. Murty, Graph Theory, Springer, New York, NY, USA, 2008.

[23] D. B. West, Introduction to Graph Theory, Prentice-Hall, Upper Saddle River, NJ, USA, 1996.

[24] S. Ou, D. Wong, F. Tian, and Q. Zhou, "Fixing number and metric dimension of a zero-divisor graph associated with a ring," 2020.

[25] J. A. Nash-Williams, "Edge-disjoint Hamiltonian circuits in graphs with vertices of high valency," in Studies in Pure Mathematics, L. Mirsky, Ed., pp. 157-183, Academic Press, London, UK, 1971. 